\title{
Kosmetische Inspektion von Glaskörpern mittels Mehrzeilen-Scantechnik
}

\author{
Karl Voth ${ }^{1}$, Matthias Hellmich ${ }^{1}$, Dr. Wolfram Acker ${ }^{2}$ \\ ${ }^{1}$ Gerresheimer Bünde GmbH, Erich-Martens-Str. 26-32, 32257 Bünde \\ k. voth@gerresheimer. com, m.hellmich@gerresheimer.com \\ ${ }^{2}$ Fachhochschule Kiel, Grenzstraße 5, 24149 Kiel \\ wolfram.acker@fh-kiel.de
}

\begin{abstract}
Zusammenfassung. Im pharmazeutischen Umfeld ist die Sicherstellung unversehrter Glasbehälter eine essentielle Herausforderung, da diese in direktem Kontakt zum Medikament stehen. Insbesondere dürfen die Behälter keine durchgehenden Risse enthalten, welche die Sterilität des Produktes gefährden. Daher ist eine 100\%-Kontrolle der Erzeugnisse notwendig. Breitet sich ein Riss entlang der optischen Achse aus, wird er für eine Kamera nahezu unsichtbar. Daher ist es zwingend notwendig, Bildaufnahmen der Prüflinge aus unterschiedlichen Perspektiven vorzunehmen. Diese Arbeit stellt eine Mehrzeilen-Scantechnik zur Digitalisierung der Glasoberfläche mit einer Matrixkamera aus mehreren Perspektiven vor. Diese Methode reduziert die Anzahl benötigter Kameras erheblich und ermöglicht weitergehende Bildanalysen, da die Aufnahme der einzelnen Bildzeilen synchron erfolgt. Diese Veröffentlichung schließt mit einem Beispiel zur Rissdetektion mittels synchroner Aufnahme mehrerer Zeilenbilder ab. Die Sensorbereiche sind derart gewählt, dass die Zeilenbilder unter unterschiedlichen Winkeln zur Glasoberfläche erfasst werden. Neben einem Zeilenbild, dass den Scheitelpunkt des Glaszylinders erfasst, werden weitere Zeilenbilder akquiriert, welche die Glasoberfläche in einem anderen Winkel erfassen. Somit stehen mehrere Bilder zur Verfügung, die sowohl separat, als auch unter Nutzung deren spatiotemporalen Zusammenhangs verarbeitet werden können.
\end{abstract}

Schlüsselwörter: Bildverarbeitung, Glasinspektion, Zeilenbilder, Rissdetektion

\section{Einführung}

Gerresheimer ist ein weltweit führender Hersteller hochwertiger Spezialprodukte aus Glas und Kunststoff für die internationale Pharma- und HealthcareIndustrie. Der Standort Bünde der Gerresheimer AG ist das Center of Excellence für vorfüllbare Glasspritzen und -karpulen.

Die Glasverarbeitung ist komplex und aufgrund der notwendigen hohen Temperaturen prozessbedingt fehleranfällig [1]. Hunderte Parameter beeinflussen die Prozessgüte und haben einen direkten Einfluss auf die Produktqualität. Dennoch müssen im pharmazeutischen Umfeld sehr hohe Qualitätsstandards eingehalten werden [2]. Die optische 100\%-Kontrolle erfolgt mit Hilfe komplexer 
Inline-Kamerasysteme, die sowohl kosmetische als auch geometrische Qualität jedes Prüflings sicherstellen [4].

In dieser Veröffentlichung wird eine hybride Bildaufnahmemethode vorgestellt, die sowohl die Aufnahme von Matrix- als auch mehrerer Zeilenbilder mit einer Kamera ermöglicht. Innerhalb eines Messzyklus kann der Betriebsmodus geändert und somit zum Beispiel neben einer kosmetischen Prüfung der Mantelfläche eines Glaskörpers auch deren geometrische Eigenschaften wie Durchmesser und Länge geprüft werden. Darüber hinaus ermöglicht der Matrix-Modus eine hochgenaue Einrichtung der Messkamera.

\section{Anforderung an die Prüfung zylindrischer Glaskörper}

Die Qualität eines Glaskörpers - insbesondere von Spritzen, Karpulen und Injektionsfläschchen - wird im pharmazeutischen Umfeld unter anderem anhand der geometrischen Maßhaltigkeit und der Unversehrtheit des Glases bestimmt. Die Vorgaben an die Geometrie ergeben sich aus DIN-EN-ISO-Normen und Kundenanforderungen. Für die Beurteilung des Glases gibt es ebenfalls Spezifikationen, welche eine minimal zulässige Fehlergröße und -ausprägung für unterschiedliche Fehlertypen wie Risse, Kratzer, Partikel, Verschmutzungen oder Luftstreifen vorschreiben. Die Grenzen der Zulässigkeit sind gegeben durch die Größe des Fehlers und dessen mögliche Folgen. Außenliegende Verschmutzungen und Kratzer können beispielsweise bis zu einer bestimmten Größe toleriert werden, da eine Verunreinigung des innen abgefüllten Medikaments ausgeschlossen ist. Im Gegensatz dazu besteht bei Rissen im Glaszylinder immer Risiko einer Kontamination des Wirkstoffs.

Prozessbedingt können solche Fehler, die im Folgenden als kosmetische Fehler bezeichnet werden, nicht ausgeschlossen werden. Die von der Firma Gerresheimer entwickelten Systeme ermöglichen dabei die Detektion von Defekten, die weit unter der Sichtbarkeit durch das menschliche Auge liegen. Um die Ausbringung in diesem Punkt dennoch ökonomisch zu halten, ist eine zweifelsfreie Klassifizierung und separate Bewertung einer jeden Fehlerklasse notwendig.

Kosmetische und strukturelle Fehler auf einer Glasoberfläche oder im Glaskörper wie Risse, Kratzer oder Verschmutzungen reduzieren die Lichtdurchlässigkeit oder brechen das Licht. Deren Prüfung erfolgt daher mittels Durchlichtanwendung. Der Prüfling wird zwischen Kamera und einer diffusen Beleuchtung platziert und der Schattenwurf der Fehler analysiert. Über mehrstufige Schwellwertverfahren werden Anomalien extrahiert und anschließend anhand ihrer morphologischen Eigenschaften beurteilt. Diese Formmerkmale wie Länge, Breite, Fläche oder Rundheit werden zur Unterscheidung von Fehlertypen hinzugezogen. So sind Partikel zum Beispiel klein und kreisähnlich, Kratzer länglich, und grobe Verschmutzungen weisen eine hohe Gesamtfläche auf, die jedoch einen eher geringen Kontrast haben kann. Im letzten Schritt wird entschieden, ob der Fehler laut Spezifikation zulässig ist, oder ob das Produkt zu verwerfen ist. Abbildung 1 zeigt übliche Fehlertypen. 
a)

b)

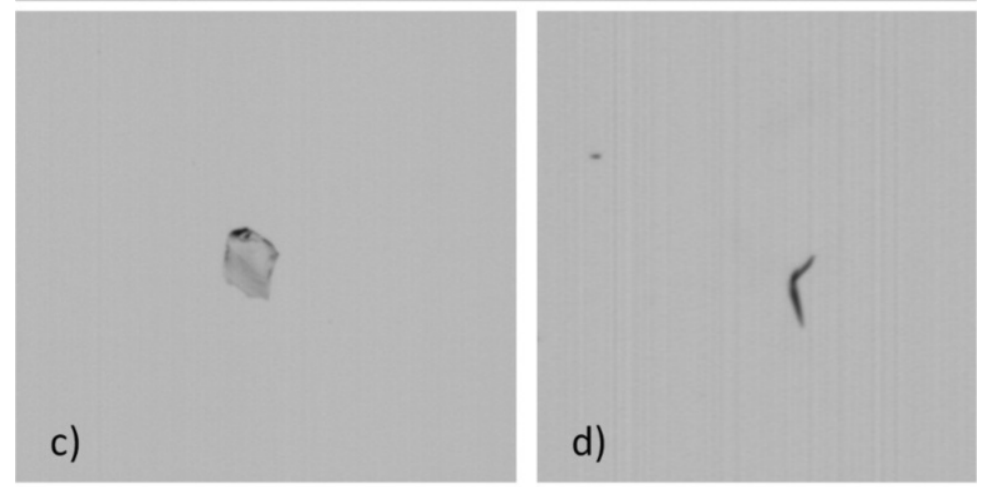

Abb. 1. Kosmetische und strukturelle Fehler im Glas. Luftstreifen (a), Kratzer (b), Glaspartikel (c) und Kerbe (d).

Eine besondere Anforderung an die Inspektionssysteme stellt die Rissdetektion dar. Breitet sich ein Riss entlang der optischen Achse aus, wird er in einer Durchlichtanwendung nahezu unsichtbar (Abbildung 2, links). Ist die Rissrichtung nicht parallel zur optischen Achse, wird der abgeschattete Bereich größer und der Fehler wird sichtbar (Abbildung 2, rechts).

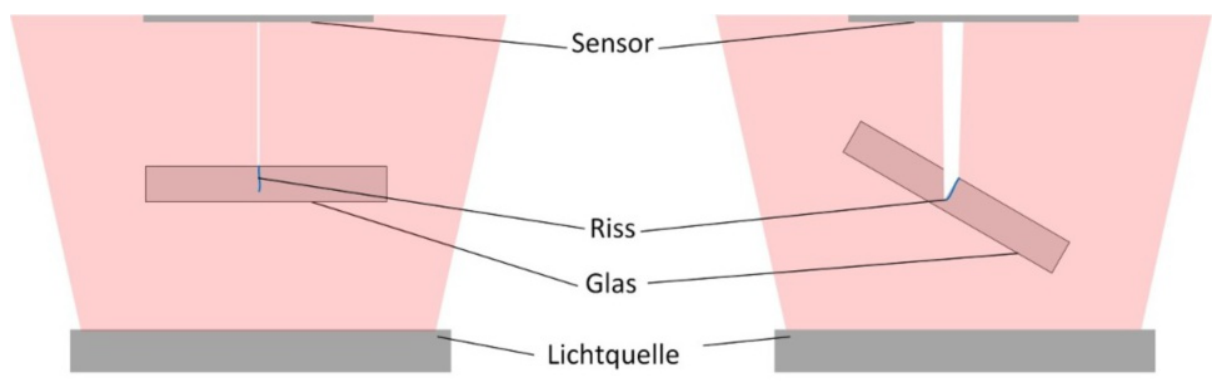

Abb. 2. Risserfassung aus mehreren Perspektiven. 
Um eine zuverlässige Detektion von Rissen im Glas zu gewährleisten, sind Bildaufnahmen aus mehreren Perspektiven erforderlich. Bei einem Glaszylinder ist der Winkel $\alpha$ am Scheitelpunkt orthogonal zur optischen Achse:

$$
\alpha=\cos ^{-1}\left(\frac{a}{r}\right) \text {. }
$$

Der Parameter a beschreibt die Auslenkung aus dem Zentrum, r gibt den Radius des Kreises an. Wird ein Matrixsensor zur Digitalisierung des Risses verwendet, ist dieser abhängig von seiner Ausdehnungsrichtung und der Position auf dem Zylinder unterschiedlich deutlich zu erkennen. D.h. je nachdem in welcher Position die Aufnahme getätigt wird, ändert sich die optische Repräsentation des Fehlers. Dies erschwert eine objektive Analyse und Bewertung. Wird ein Zeilenbild aus einer einzigen Perspektive erstellt, kann der Fehler nahezu unsichtbar werden, wie Abbildung 3 a) zeigt.

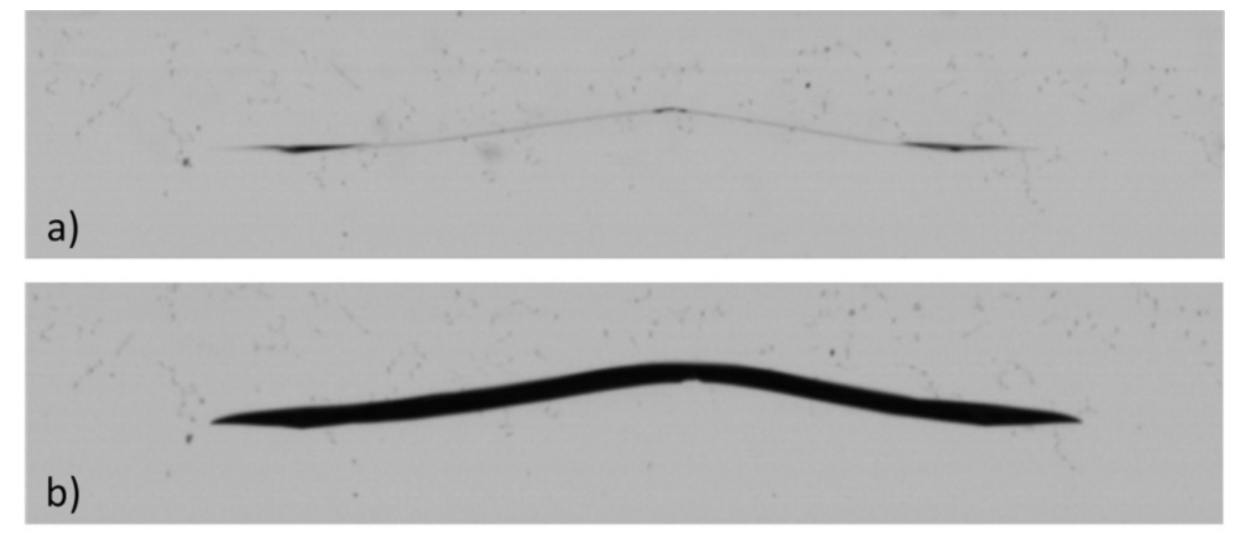

Abb. 3. Optische Repräsentation eines Risses aus zwei Perspektiven. Die Vorzugsrichtung des Fehlers beträgt bei a) ca. $90^{\circ}$ zur optischen Achse, bei b) ca. $60^{\circ}$ zur optischen Achse.

Um sicherzustellen, dass Risse zuverlässig detektiert werden, sind somit Zeilenaufnahmen aus mehreren Perspektiven durchzuführen. Ausgehend davon, dass sowohl Radial- als auch Axialrisse auftreten können, sind insgesamt vier Aufnahmen zu erfassen. Dies hat hohe Anschaffungs- und Wartungskosten zur Folge. Nachfolgend wird ein Ansatz vorgestellt, der die Aufnahme mehrer Zeilenbilder aus vielen Bildern einer Matrixkamera ermöglicht.

\section{Realisierung der Mehrzeilen-Scantechnik mit einer Matrixkamera}

Neben klassischen Zeilen- und Matrixkameras werden sogenannte „Time Delay and Integration" (TDI) -Kameras im Umfeld der industriellen Bildverar- 
beitung eingesetzt [5]. Das Verhalten dieser Kameras ähnelt dem einer Zeilenkamera, die Bildsensoren beinhalten allerdings mehrere Zeilen. Wenn sich ein Objekt gegenüber einer Kamera gleichmäßig bewegt, kann aufgrund des spatiotempralen Zusammenhangs die Integrationszeit erhöht werden. Das heißt, es wird nicht ausschließlich eine Zeile, sondern mehrere Zeilen nacheinander belichtet. So kann bei schwachen Lichtverhältnissen oder einer schnellen Bewegung des Objektes der Signal- Rauschabstand erhöht und somit die Bildqualität signifikant verbessert werden. Üblicherweise wird dem Inspektionssystem die über mehrere Zeilen hinweg integrierte Aufnahme zur Verfügung gestellt. Eine Entnahme mehrerer Zeilenaufnahmen ist in der Regel nicht möglich. Darüber hinaus liegt die Anzahl der Zeilen auf dem Sensor deutlich unter der Zeilenanzahl von Matrix-Sensoren mit vergleichbarer Sensorbreite. Eine hybride Verwendung als Matrix- und Zeilenkamera ist somit nur eingeschränkt möglich. Daher wird die Mehrzeilen-Scantechnik nicht mit TDI- sondern modernen Matrix-Bildsensoren realisiert, welche ein schnelles Auslesen von ROIs (Region of Interest) ermöglichen [6].

Einige Sensoren unterstützen auch das parallele Auslesen mehrerer voneinander unabhängiger Sensorregionen. Diese Eigenschaft kann genutzt werden, um simultan mehrere Zeilenbilder zu erfassen. Zu diesem Zweck werden auf einem Matrixsensor mehrere Zeilen als einzelne ROIs festgelegt. Durch diese Vorgehensweise entsteht eine hohe Anzahl von Einzelbilden, aus denen mehrere Zeilenbilder abgeleitet werden können. Jedes Einzelbild wird separat an die Bildverarbeitungseinheiten übertragen und dort zu Zeilenbildern zusammengesetzt. Diese Aufnahmemethode hat einerseits die Einschränkung, dass die Zeilen sequentiell übertragen werden. Somit fliefst in die maximal realisierbare Zeilenfrequenz neben der Belichtungs- und Auslesezeit auch die Übertragungszeit. Diese hängt vom Kommunikationsstandard und -medium ab. Auch ist die Belichtungszeit um mindestens die übertragungszeit kleiner als die Zeilenzeit zu wählen:

$$
t_{\text {Zeile }}>t_{\text {Belichtung }}+t_{\text {Auslesen }}+t_{\text {Uebertragung }}
$$

Darüber hinaus ändert sich auch die optische Repräsentation und optische Auflösung vor allem bei Verwendung entozentrischer Objektive abhängig von der Position der Zeile auf dem Sensor.

Andererseits können mehrere Zeilenbilder mit einer Matrixkamera erfasst werden. Die Position der Zeilen kann frei gewählt werden. Außerdem ist keine Synchronisierung der Zeilenbilder notwendig, da die Aufnahme simultan erfolgt. Dies ermöglicht die Nutzung spatiotemporaler Zusammenhänge bei der Fusion der Zeilenbilder. Das Zusammensetzen der Aufnahmen erfolgt in fieldprogrammable gate arrays (FPGAs), so dass die Rechenkapazität des Auswerterechners nicht beeinflusst wird.

Durch eine Rekonfiguration der Kamera während des Inspektionszyklus kann zwischen Zeilen- und Matrixmodus gewechselt werden. Dadurch kann zum einen die Ausrichtung der Kameras wesentlich vereinfacht werden. Die Pose, Schärfe und die Zeilenpositionen können im Vollbild justiert und überwacht werden. Zum anderen können zusätzliche Inspektionen in Matrixbildern erfolgen. Im Falle von 
Glaszylindern sind es zum Beispiel geometrische Merkmale wie Durchmesser oder Rohrlänge. Auch die Zeilenpositionen können mitgeführt werden, falls die Positionierung vor der Kamera unzureichend ist.

Durch den Betrieb als hybride Kamera für Mehrzeilen- und Matrixaufnahmen können Anschaffungs-, Einrichtungs-, Wartungskosten, Aufwand für mechanische und elektrische Integration, und Fehleranfälligkeit signifikant reduziert werden. Darüber hinaus reduziert sich die Anzahl der benötigten Handhabungssysteme. Eine Inspektion des Glaszylindermantels erfordert die temporäre Entnahme des Prüflings aus dem Transportsystem. Der Glaskörper wird ausgehoben, rotiert und anschließend wieder abgelegt (s. Abbildung 4).

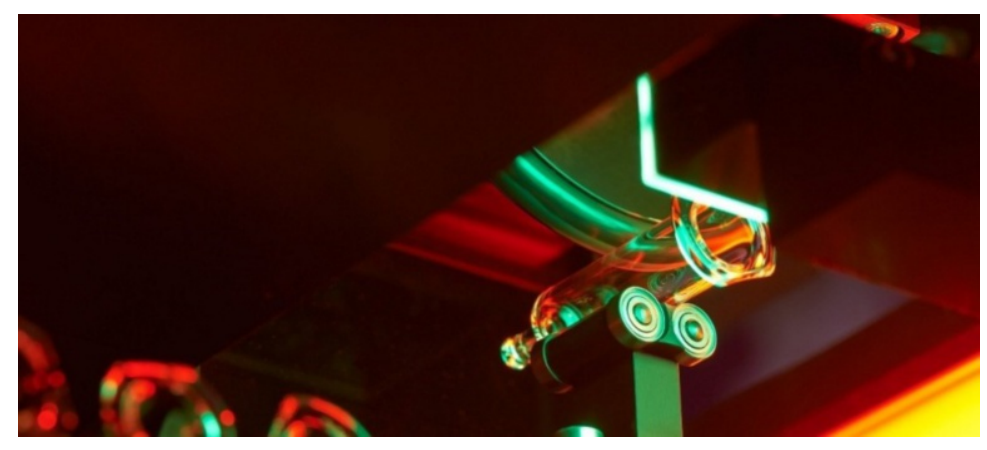

Abb. 4. Handhabungseinheit für die Inspektion einer Glasspritze. Unterhalb der Spritze befindet sich die Aushebeeinheit. Das Rad oberhalb des Prüflings versetzt diesen in Rotation.

Die Reduktion der Handhabungssysteme verringert die Komplexität der Anlage und kommt auch der Produktqualität zugute, da jede zusätzliche Produktberührung ebenfalls eine potentielle Ursache von Beschädigungen und Verschmutzungen ist.

\section{Rissdetektion im Glas mittels Mehrzeilen-Scantechnik}

Durch die simultane Aufnahme mehrerer Zeilenbilder können zusätzliche Informationen durch eine Fusion dieser extrahiert werden. Wie in Abbildung 2 bereits skizziert, ändert ein Riss seine optische Repräsentation, wenn er aus unterschiedlichen Perspektiven erfasst wird. Andere Fehler wie zum Beispiel Partikel oder Kratzer weisen die Dreidimensionalität nicht in dieser Ausprägung auf. Dieses Merkmal kann nun dazu genutzt werden, um einen Riss von anderen Fehlern zu unterscheiden. Abbildung 5 zeigt die Aufnahme eines Risses mit der MehrzeilenScantechnik. Neben dem Riss ist auch ein Partikel zu sehen, der rot eingekreist ist. Darüber hinaus sind kleine Kratzer über das gesamte Bild verteilt. Der Riss dringt im mittleren Bereich tief in das Glas ein. Nach außen hin nimmt die Risstiefe ab. 

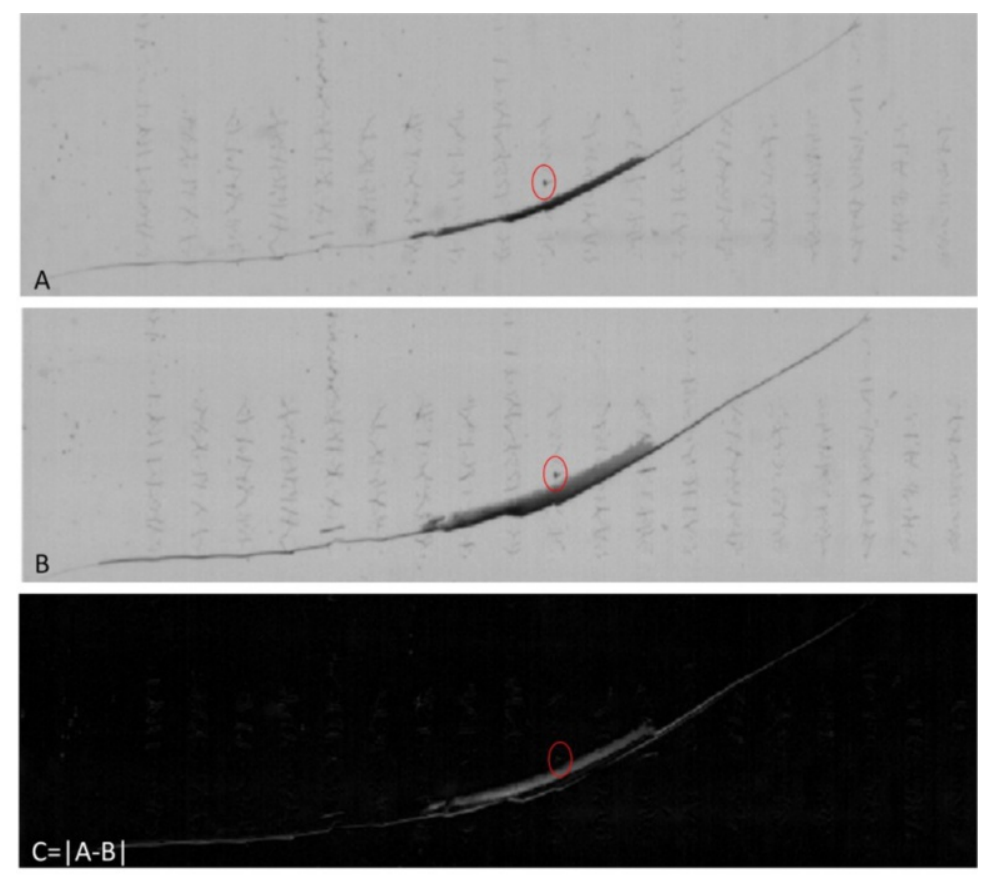

Abb. 5. Aufnahme eines Risses mit einer Mehrzeilen-Scankamera und deren pixelweise Differenz. Der rot umkreiste Partikel verschwindet in dem Differenzbild.

Um den Riss eindeutig segmentieren zu können, wird im ersten Schritt die räumliche Distanz zwischen der optischen Repräsentation des Risses eliminiert. Dazu werden die Umfangsgeschwindigkeit und der Abstand zwischen den Zeilen auf dem Matrixsensor genutzt. Anschließend erfolgt eine pixelweise Differenzbildung der Bilder A und B durchgeführt.

Während der Riss nach wie vor deutlich zu erkennen ist, sinkt der Kontrast anderer Fehler. Der rot eingekreiste Partikel ist zum Beispiel nahezu vollständig verschwunden. Weitere Fehler, wie zum Beispiel einige tiefe Kratzer sind nach wie vor zu erkennen. Dies liegt daran, dass diese Fehler ebenfalls ins Glas eindringen. Im nächsten Schritt kann der Riss mit Hilfe von histogrammbasierten Schwellwertverfahren segmentiert werden. Da ein Riss ins Glas eindringt und sich nicht ausschließlich an der Oberfläche befindet, kann er über die Fehlerbreite im Referenzbild eindeutig identifiziert werden.

\section{Zusammenfassung und Ausblick}

Die Arbeit zeigt eine Möglichkeit zur Verwendung einer Matrixkamera für die synchrone Aufnahme sowohl mehrerer Zeilenbilder als auch Matrixbilder innerhalb eines Messzyklus auf. Mit dieser Technik können zum Beispiel Risse im 
Glaszylinder anhand ihrer Tiefe identifiziert werden, da sie aus mehreren Perspektiven digitalisiert werden. Darüber hinaus ermöglicht diese Aufnahmemethode eine Reduktion der Kameraanzahl, Senkung der Kosten für mechanische und elektronische Einbindung und Vereinfachung der Einrichtung und Wartung von Inspektionssystemen. Zukünftig werden weitere Fusionsansätze untersucht und zusätzliche Eigenschaften der Fehler wie zum Beispiel die Messung der Risstiefe implementiert.

\section{Literatur}

1. Delgado Carranza, José de Jesús, Guzmán, T. R. und Garcia, A. M.: Verfahren zum Herstellen von Spritzen. DE000019955791A1 (1999)

2. Rimkus, F. R. und Stieneker, F.: Pharmazeutische Packmittel. Primär-/ Sekundärpackmittel, Qualität, regulatorische Anforderungen. ECV - Editio-Cantor-Verl., Aulendorf, Württ (2013)

3. Dastis, H.: Prozessoptimierung in der pharmazeutischen Röhrenglasweiterverarbeitung. Dissertation, Technische Universität Bergakademie Freiberg, Freiberg, (2007)

4. Voth K., Hellmich M. und Acker W.: Prozessintegrierte Bildverarbeitung im pharmazeutischen Umfeld. Springer Vieweg Berlin Heidelberg, Heidelberg (2016)

5. H. -. Wong, Y. L. Yao und E. S. Schlig: TDI charge-coupled devices: Design and applications. IBM Journal of Research and Development (1992)

6. Hellmich M. und Acker W.: Mehrzeilen-Scantechnik. Gerresheimer Bünde GmbH, EP2851677A1 (2015)

Open Access Dieses Kapitel wird unter der Creative Commons Namensnennung 4.0 International Lizenz (http://creativecommons.org/licenses/by/4.0/deed.de) veröffentlicht, welche die Nutzung, Vervielfältigung, Bearbeitung, Verbreitung und Wiedergabe in jeglichem Medium und Format erlaubt, sofern Sie den/die ursprünglichen Autor(en) und die Quelle ordnungsgemäß nennen, einen Link zur Creative Commons Lizenz beifügen und angeben, ob Änderungen vorgenommen wurden.

Die in diesem Kapitel enthaltenen Bilder und sonstiges Drittmaterial unterliegen ebenfalls der genannten Creative Commons Lizenz, sofern sich aus der Abbildungslegende nichts anderes ergibt. Sofern das betreffende Material nicht unter der genannten Creative Commons Lizenz steht und die betreffende Handlung nicht nach gesetzlichen Vorschriften erlaubt ist, ist für die oben aufgeführten Weiterverwendungen des Materials die Einwilligung des jeweiligen Rechteinhabers einzuholen. 